\title{
THE EMBEDDING OF TWO-SPHERES IN THE FOUR-SPHERE
}

\author{
BY HERMAN GLUCK ${ }^{1}$
}

\author{
Communicated by A. W. Tucker, June 23, 1961
}

1. Introduction. An embedding $h$, of $S^{2}$ in $S^{4}$ will be called smooth if $h$ can be extended to an embedding of $S^{2} \times E^{2}$ in $S^{4}$, where $E^{2}$ is the open unit disc in the plane and $S^{2}$ is identified with $S^{2}$ ×origin. The embedding $h$ will be called semi-linear if $h$ is a simplicial homeomorphism of some rectilinear subdivision of the boundary of a 3-simplex with a subcomplex of some rectilinear subdivision of the boundary of a 5 -simplex. If $S^{2}$ is semi-linearly embedded in $S^{4}$ and $v$ is a vertex of $S^{2}$, then the link of $v$ on $S^{2}$ is a simple closed curve, while the link of $v$ in $S^{4}$ is a three-sphere. If this simple closed curve is unknotted in this three-sphere, then $S^{2}$ is said to be locally flat at $v$. If $S^{2} \subset S^{4}$ is locally flat at all of its vertices, then it is said to be locally flat. Noguchi has shown in [1, Theorem 3] that every locally flat semilinear $S^{2}$ in $S^{4}$ is smoothly embedded, and that its regular neighborhoods are homeomorphic to $S^{2} \times E^{2}$.

Let $S^{2}$ have a neighborhood $S^{2} \times E^{2}$ in $S^{4}$. Let $E^{2 \prime}$ be the open disc of radius $1 / 2$. Then $A^{4}=S^{4}-\left(S^{2} \times E^{2 \prime}\right)$ is an orientable four-manifold whose single boundary component is homeomorphic to $S^{2} \times S^{1}$. $A^{4}$ is called an exterior of $S^{2}$ in $S^{4}$. If the boundary component of $A^{4}$ is removed, the remaining open manifold is clearly homeomorphic to $S^{4}-S^{2}$. I do not know whether two different exteriors of a smooth $S^{2}$ in $S^{4}$ are necessarily homeomorphic. In the locally flat semi-linear case, however, we can define the exterior to be the complement in $S^{4}$ of any open regular neighborhood of $S^{2}$. Then the exterior is welldefined, for one can always find a semi-linear homeomorphism of $S^{4}$ onto itself taking one regular neighborhood of $S^{2}$ onto any other (see $[2])$.

Two pairs $\left(S^{4}, S^{2}\right)$ and $\left(S^{4 \prime}, S^{2 \prime}\right)$ are said to be equivalent (or homeomorphic) if there is a homeomorphism $h$ of $S^{4}$ onto $S^{4 \prime}$ which carries $S^{2}$ onto $S^{2 \prime}$. We can then in any case ask how strong an invariant of the pair $\left(S^{4}, S^{2}\right)$ we get by considering the topological type of an exterior $A^{4}$ of $S^{2}$ in $S^{4}$. In other words, how many nonequivalent smooth embeddings of $S^{2}$ in $S^{4}$ can have an exterior homeomorphic to $A^{4}$ ?

1 The author is a National Science Foundation Predoctoral Fellow and wishes to thank Professor Ralph Fox for his help in the preparation of this paper. Detailed proofs of the results stated here are contained in the author's thesis. 
THEOREM 1. There are at most two nonequivalent smooth embeddings of a two-sphere in the four-sphere with a given exterior.

The proof will be outlined in the following sections and will appear in detail elsewhere. While it is easy to show that an exterior is a complete invariant for certain classical embeddings of $S^{2}$ in $S^{4}$, the question of whether this is always so for all smooth embeddings is still open.

Let $D^{2}$ be the closed unit disc in the plane and $S^{1}$ its boundary. Then every smooth embedding of $S^{2}$ in $S^{4}$ with an exterior homeomorphic to $A^{4}$ can be obtained, up to homeomorphism, by identifying the boundary $S^{2} \times S^{1}$ of $S^{2} \times D^{2}$ with the boundary $S^{2} \times S^{1}$ of $A^{4}$ via some homeomorphism $h$ of $S^{2} \times S^{1}$. Furthermore, it is easily seen that the pairs obtained by using two isotopic homeomorphisms of $S^{2} \times S^{1}$ are equivalent. Thus instead of considering the group of all homeomorphism of $S^{2} \times S^{1}$, we can factor out the normal subgroup consisting of those homeomorphisms which are isotopic to the identity. Under the compact-open topology, this subgroup is the arcwise connected component of the identity. The resulting quotient group, $\mathcal{H C}\left(S^{2} \times S^{1}\right)$, is called the group of homeomorphism types of $S^{2} \times S^{1}$. It has also been called the homeotopy group of $S^{2} \times S^{1}$ (see [3]).

It is possible that some adjunctions of $S^{2} \times D^{2}$ with $A^{4}$ may not even give $S^{4}$ for the adjunction space, while other nonisotopic identifications may nevertheless yield equivalent pairs. In any case, a natural beginning for the proof of Theorem 1 is to compute the group $\mathfrak{H C}\left(S^{2} \times S^{1}\right)$.

2. The group of homeomorphism types of $S^{2} \times S^{1}$. A homeomorphism of $S^{2} \times S^{1}$ induces an automorphism of $H_{1}\left(S^{2} \times S^{1} ; Z\right) \approx Z$ and an automorphism of $H_{2}\left(S^{2} \times S^{1} ; Z\right) \approx Z$, each of which depends only on the isotopy class of the homeomorphism. Thus we get a homomorphism $\phi: \mathfrak{H C}\left(S^{2} \times S^{1}\right) \rightarrow Z_{2} \oplus Z_{2}$. Let $r: S^{2} \rightarrow S^{2}$ be the antipodal map and $s: S^{1} \rightarrow S^{1}$ the map induced on the unit circle by complex conjugation. Let $\mathcal{F C}^{\prime} \approx Z_{2} \oplus Z_{2}$ be the subgroup of $\mathcal{F}$ consisting of the isotopy classes of the maps $(1,1),(1, s),(r, 1),(r, s)$. Let $\rho$ be the isomorphism of $Z_{2} \oplus Z_{2}$ with $\mathcal{H C}^{\prime} \subset \mathcal{H C}$ determined by the condition $\phi \rho=1$.

The main result is that the kernel of $\phi$ is isomorphic to $Z_{2}$. But a normal subgroup of order two is central. This and the fact that $\phi$ splits implies that $\mathfrak{H} \approx Z_{2} \oplus Z_{2} \oplus Z_{2}$.

TheOREM 2. $\operatorname{HC}\left(S^{2} \times S^{1}\right) \approx Z_{2} \oplus Z_{2} \oplus Z_{2}$.

As just remarked, it remains to be shown that ker $\phi \approx Z_{2}$. The proof proceeds in seven stages, during which we think of $S^{2}$ as the unit 
sphere in three-space and of $S^{1}$ as the space of real numbers modulo 1. Denote by 1 the identity homeomorphism of $S^{2} \times S^{1}$. Let $\Phi_{\alpha}$ denote a rotation of $S^{2}$ about a diameter through the north and south poles through an angle $2 \pi \alpha$ in some fixed direction. Define the homeomorphism $T$ of $S^{2} \times S^{1}$ by

$$
T(x, t)=\left(\Phi_{t}(x), t\right) .
$$

By the genus of a homeomorphism of $S^{2} \times S^{1}$ we shall mean the image of its isotopy class under $\phi$. Thus the kernel of $\phi$ consists of the isotopy classes of the homeomorphisms of genus $(1,1)$. Both 1 and $T$ are of genus $(1,1)$. We show then that any homeomorphism of $S^{2} \times S^{1}$ of genus $(1,1)$ is isotopic either to 1 or to $T$. Let $C$ be a small circle on $S^{2}$ about the north pole. In the first five stages we deform an arbitrary homeomorphism, $h$, of genus $(1,1)$ until $h /\left(S^{2} \times 0\right)$ $\cup\left(C \times S^{1}\right)$ is either the identity or else coincides with $T /\left(S^{2} \times 0\right)$ $\cup\left(C \times S^{1}\right)$. In stage six, we deform $h$ into either 1 or $T$. The final stage uses the homotopy classification of maps of $S^{2} \times S^{1}$ into $S^{2}$ given by Pontrjagin, [4], to show that $T$ is not homotopic to 1 ; a fortiori, not isotopic to 1 .

CoRollary to Theorem 2. Two homeomorphisms of $S^{2} \times S^{1}$ are isotopic if and only if they are homotopic.

3. Two-spheres in the four-sphere with a given exterior. Theorem 2 tells us that there are at most eight nonequivalent smooth embeddings of $S^{2}$ in $S^{4}$ with a given exterior.

The extendibility of a homeomorphism of $S^{2} \times S^{1}$ to a homeomorphism of $S^{2} \times D^{2}$ depends only on the isotopy class of the homeomorphism. Thus, if the isotopy class of the homeomorphism is contained in the subgroup $\mathfrak{H C}^{\prime}$ of $\mathfrak{H}$, then the homeomorphism can be extended to a homeomorphism of $S^{2} \times D^{2}$. We then get the following theorem, in which $A^{4}$ denotes an exterior of $S^{2}$ in $S^{4}$.

Theorem 3. Let the pair $\left(M^{4}, S^{2}\right)$ be obtained by joining $S^{2} \times D^{2}$ to $A^{4}$ by the homeomorphism $f$ of $S^{2} \times S^{1}$. Similarly, let the pair $\left(N^{4}, S^{2}\right)$ be obtained by joining $S^{2} \times D^{2}$ to $A^{4}$ by the homeomorphism $g$ of $S^{2} \times S^{1}$. If the isotopy class of $\mathrm{g}^{-1} f$ is an element of $\mathrm{JC}^{\prime}$, then $\left(M^{4}, S^{2}\right)$ is homeomorphic to $\left(N^{4}, S^{2}\right)$.

But $\mathfrak{H C}^{\prime}$ is of index two in $\mathfrak{H C}$, from which Theorem 1 follows.

4. Concluding remarks. If we join the boundary of $S^{2} \times D^{2}$ to that of $A^{4}$ by the homeomorphism $T$ of $S^{2} \times S^{1}$, we get a manifold $M^{4}$ and a distinguished smooth two-sphere $S^{2}$ in $M^{4}$. The original pair $\left(S^{4}, S^{2}\right)$ 
and the new pair $\left(M^{4}, S^{2}\right)$ give the only possible smooth embeddings of a two-sphere in a four-manifold with an exterior homeomorphic to $A^{4}$. The following assertions hold:

(4.1) $M^{4}$ is a homotopy four-sphere.

(4.2) If the two-sphere $S^{2} \times 0$ on the boundary of $A^{4}$ is itself the boundary of a three-sphere with handles, "nicely" situated in $A^{4}$, then the pair $\left(M^{4}, S^{2}\right)$ is homeomorphic to the original pair $\left(S^{4}, S^{2}\right)$.

(4.3) In particular, any two-sphere in $S^{4}$ obtained by spinning a tame knot in three-space (see $[5 ; \mathbf{6}]$ ) is uniquely determined by the topological type of its semi-linear exterior (the complement in $S^{4}$ of any open regular neighborhood of the twosphere).

(4.4) Modulo the conjecture that two homeomorphisms of $S^{n} \times S^{1}$ are isotopic if and only if they are homotopic, Theorems 1 , 2 and 3 also hold for smooth embeddings of $S^{n}$ in $S^{n+2}$.

\section{REFERENCES}

1. H. Noguchi, On regular neighborhoods of 2-manifolds in 4-Euclidean space. I, Osaka Math. J. vol. 8 (1956) pp. 225-242.

2. J. H. C. Whitehead, Simplicial spaces, nuclei and m-groups, Proc. London Math. Soc. Ser. 2, vol. 45 (1938) pp. 243-327.

3. G. S. McCarty, Jr., Homeotopy groups, Abstract 61T-48, Notices Amer. Math. Soc. vol. 8 (1961) p. 67.

4. L. Pontrjagin, $A$ classification of mappings of the three-dimensional complex into the two-dimensional sphere, Recueil Mathématique vol. 9 (1941) pp. 331-359.

5. E. Artin, Zur Isotopie zweidimensionaler Flächen in $R_{4}, \mathrm{Abh}$. Math. Sem. Univ. Hamburg vol. 4 (1925) pp. 174-177.

6. J. J. Andrews and M. L. Curtis, Knotted 2-spheres in the 4-sphere, Ann. of Math. vol. 70 (1959) pp. 565-571.

Princeton University 